\title{
Cellular Senescence by the Epigenetic Regulators Inhibitor of Growth
}

Thanakorn Pungsrinont and Aria Baniahmad

Professor, Institute of Human Genetics, Jena University Hospital, Germany

*Corresponding author: Aria Baniahmad, Institute of Human Genetics, Jena University Hospital Kollegiengasse 10, 07743 Jena, Germany, Tel: +49-3641-935524; Fax: +49-3641-934706; E-mail: aria.baniahmad@med.uni-jena.de

Received date: December 18, 2015; Accepted date: January 18, 2016; Published date: January 25, 2016

Copyright: () 2015 Pungsrinont and Baniahmad. This is an open-access article distributed under the terms of the Creative Commons Attribution License, which permits unrestricted use, distribution, and reproduction in any medium, provided the original author and source are credited.

\begin{abstract}
The epigenetic regulatory tumor suppressor, INhibitor of Growth 1 (ING1), obtained more focus since it has been suggested as one of the aging-related candidate genes among healthy elderly individuals. ING1 belongs to the ING family proteins characterized by a plant homeodomain (PHD), which is important for recognizing and binding to histone marks, thus allowing ING to regulate genes expression through histone modification and chromatin changes. Interestingly, the PHD of ING proteins is highly conserved among species between mammals, insects and plants. The ING factors regulate the program of cellular senescence and DNA repair, which are suggested to have a protective role in inhibiting cancer cells proliferation. Here, we provide an insight into the functional role of ING factors in development and tumor cells.
\end{abstract}

Keywords: ING; Senescence; Cancer; Epigenetics; Aging; PHD; Evolution

\section{Abbreviations: \\ H3K4me3, histone 3 trimethylation at lysine 4; HAT, histone acetyltransferase; HDAC, histone deacetylase; HMT, histone methyltransferase; ING, Inhibitor of growth; KD, knockdown; KO, knockout; PHD, plant homeodomain.}

\section{Introduction}

Epigenetic and genetic factors are suggested to be involved in the aging process [1-3]. Indeed, aging research on various model organisms like Caenorhabditis elegans or Drosophila melanogaster improved our understanding of genomic, epigenetic and proteomic aspects regarding the lifespan of these organisms [2-5]. Specific set of genes or genetic loci that are related to longevity and aging are being analyzed in these model systems. Genetic and epigenetic factors appear to have significant influence also on human longevity, since the heritability of human lifespan was estimated in a range of $20-30 \%$ in many studies [6-10]. Unlike genetics, epigenetics refers to "functional changes of the genome without changing the DNA sequence". This includes chromatin changes and remodeling, which in general is triggered by factors that promote or remove histone modifications and regulate exchange of histone variants $[11,12]$. However, the underlying mechanisms linking epigenetics to aging are poorly understood. One reason is the fact that aging is associated with a variety of human disorders, which includes cancer [13].

Interestingly, the gene encoding the epigenetic regulator tumor suppressor, inhibitor of growth 1 (ING1) has been suggested to be one of the aging-related candidate genes among 47 healthy individuals at the age of 85 years or older [14]. Within this cohort, no aging-related diseases such as cancer, cardiovascular disease, pulmonary disease, diabetes, or Alzheimer disease have been diagnosed.

The ING tumor suppressors are localized in the nucleus and directly associated with chromatin regulation and control of gene expression
$[15,16]$. ING factors control various cellular pathways which include cell cycle control, DNA repair and two tumor protective pathways: apoptosis and cellular senescence that both seems to be important pathways for tumor suppression.

In general, the Hayflick limit suggests a limited cell division potential of primary cells that is also termed replicative lifespan [17]. Cells having approached the replicative lifespan are metabolically active, exhibit a changed morphology, and are also termed to be cellular senescent. Cellular senescence occurs naturally in vivo during normal development and is involved in embryonic patterning [18]. Also during tumorigenesis, the pre-malignant tumors exhibit high levels of senescent cells in vivo [19]. During further steps of tumor progression, the level of senescent cells declines, suggesting that malignant tumor cells evade from the cellular senescence pathway and from the other anti-tumor pathway, apoptosis. It is therefore suggested that during tumorigenesis, tumor cells escape from the cellular senescence pathway and undergo selection to evolve and develop into malignant cancer.

Interestingly, cellular senescence is a cellular pathway that is characterized by an irreversible cell cycle arrest that is mostly induced by replicative lifespan or cellular stress and therefore is suggested to act against cancer malignancy $[19,20]$. However, cellular senescence can be either detrimental or beneficial, depending on the physiological context and situation. Cellular senescence can disrupt normal tissue structures and functions, but on the other hand, cellular senescence is an effective mechanism to suppress cancer cells proliferation [19-21]. Notably, in primary human cells the ectopic expression of either ING1 or ING2 alone is sufficient to inhibit cell proliferation by inducing cellular senescence as an underlying mechanism [22,23].

Among the ING family members, the human ING1 and ING2 are two closely related proteins that share high identity and homology in amino acid sequence [24], indicating that they exhibit similar tumor suppressive functions. The most widely expressed isoform of ING1 and ING2 are ING1b and ING2a, respectively. Most studies support their role as tumor suppressors as their expression is often found to be decreased or lost in many human tumors [25-29]. The loss or 
reduction of ING1 and/or ING2 expression could be the result of misregulation of transcription factors or gene inactivation mechanisms, and since their loss often occurs at an early stage of tumor development [30-32], it suggests that decreasing ING1 or ING2 expression in pre-malignant tumors contributes at an early stage to malignant tumorigenesis.

To analyze their functional role in tumor cells, many overexpression studies revealed that either ING1 or ING2 expression result in tumor growth inhibition $[22,23,33,34]$ and accordingly knockdown (KD) or knockout $(\mathrm{KO})$ result in an enhanced tumor proliferation [33,35-37]. These studies provide an insight to understand the functions of ING1 and ING2 to regulate cell growth. The molecular pathways are discussed below.

\section{ING proteins as epigenetic regulatory tumor suppressors induce cellular senescence through their PHD}

A tumor suppressor gene encodes for a protein that suppresses tumor growth. The ING family of genes and the corresponding encoded proteins were originally identified in 1996 [33], and later characterized as candidates for tumor suppressors because they are involved in many processes such as cell growth, apoptosis, cellular senescence, migration, and DNA repair $[32,38]$. The ING proteins are characterized by a well-conserved carboxyl-terminal region that contains a plant homeodomain (PHD) [24]. The histone binding and modification is an interesting ability of ING proteins. The PHD selectively binds preferentially to trimethylated lysine 4 of the histone 3 (H3K4me3), which is present in nucleosomes of transcriptional active genes at promoters and downstream of transcription start sites $[39,40]$, thus linking the ING proteins to epigenetic regulation [16].

Although the PHD domain of both ING1 and ING2 binds to the activating mark of the histone modification (H3K4me3), it was a surprise that ING1 and ING2 interact with a histone deacetylase (HDAC) complex. The amino-terminus of ING1 and ING2 directly interacts with the mSin3a/HDAC1-2/SAP30 complex that leads to gene silencing $[16,39,41]$. This suggests that ING tumor suppressors, if recruited, may counteract and inhibit some active gene loci. For example, it has been described that $\mathrm{H} 3 \mathrm{~K} 4 \mathrm{me} 3$ is required for ING2 binding at the cyclin D1 promoter and that cyclin D1 expression is transcriptionally inactivated by the $\mathrm{mSin} 3 \mathrm{a} / \mathrm{HDAC} 1$ complex [42]. Cyclin D1 expression is controlled by E2F factors that are regulated by the retinoblastoma protein (pRb). Thus, these findings link the ING protein to the induction of cellular senescence through inactivation of pRb by reducing the activity of cyclin D1-cyclin-dependent kinase 4 (CDK4) complex (Figure 1).

Interestingly, ING1 and ING2 are also complexed with histone methyltransferase (HMT) [39,43]. The HMT activity can methylate both histones $\mathrm{H} 1$ and $\mathrm{H} 3$ at the amino-terminal residues. The ING2associated HMT activity seems to methylate mono- and dimethylation of histone $\mathrm{H} 3$ at lysine 4 [43]. This finding suggests that ING factors recognize and modify histone marks with the PHD region that is required for chromatin association to active chromatin sites.

Interestingly, ING1 and ING2 also interact with the transcriptional coactivator p300, which has an intrinsic histone acetyltransferase (HAT) activity $[23,44]$. This interaction leads to epigenetic changes by hyperacetylation of histones, which seems to link ING function rather towards DNA repair pathway. In line with this, it has been shown that ING1 can interact with Gadd45a $[45,46]$, and both ING1b and ING2a with PCNA $[47,48]$ to mediate nucleotide excision repair.
Furthermore, ING1 and ING2 have been reported to directly bind to the promoter of CDK inhibitors, p16 and p21, respectively [49,50]. In line with this, ING1 up-regulates p16 transcription via p300 HAT activity and induces cellular senescence (Figure 1), while the underlying mechanism that ING2 positively regulates p21 transcription remains unclear.

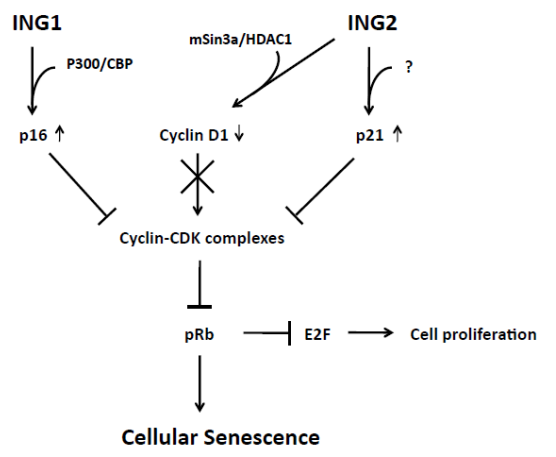

Figure 1: Model of cellular senescence induction by the ING proteins as epigenetic regulators.

ING1 and $\mathrm{p} 300 / \mathrm{CBP}$ interact with $p 16$ promoter and positively regulate its transcription. As cyclin-dependent kinase (CDK) inhibitor, p16 inhibits the activity of cyclin-CDK complexes, thus, preventing the phosphorylation of retinoblastoma protein $(\mathrm{pRb})$ keeping it active. Active $\mathrm{pRb}$ remains bound with $\mathrm{E} 2 \mathrm{~F}$, a pro-proliferative transcription activator, and suppresses its activity. This leads therefore to the inhibition of cell proliferation and triggers the cellular senescence pathway. ING2 interacts with the promoter of $p 21$, another CDK inhibitor, and activate $p 21$ transcription via an unknown mechanism. On the other hand, ING2 can bind to cyclin D1 promoter via its PHD to the histone mark of $\mathrm{H} 3 \mathrm{~K} 4 \mathrm{me} 3$. The recruitment of mSin3a/HDAC1 complex suppresses the transcription of the cyclin D1 gene. Both upregulation of p21 and down-regulation of cyclin D1 reduce the cyclinCDK complexes activity, leading to cellular senescence.

These suggest that ING tumor suppressors can either activate or inhibit the target genes transcription, and that ING proteins change their signaling dependent on the environment that induce a specific interaction with different factors. This may subsequently induce INGdistinct pathways, e.g. cellular senescence or DNA repair. Indeed, both functions are important for tumor suppression.

Evidence suggests that ING1 is involved in regulating the replicative lifespan, as the knockdown of ING1b expression results in increased number of replications [35]. In addition the expression of ING1b was found to be 8 to 10 times higher in senescent cells compared to young proliferating human fibroblasts [35]. Although the induction of cellular senescence may be a multifactorial process, data suggest that the overexpression of only one of the tumor suppressors ING1 or ING2 leads to the induction of cellular senescence [22,23]. This indicates an overlapping functional role of ING1 and ING2. The overexpression of ING1b in non-tumorigenic primary human fibroblasts resulted in growth arrest with the induction of cellular senescence [22]. Similarly, the expression of ING2a in early passage of primary human fibroblasts also showed cellular senescence inducing capability [23]. The functional consequences of ING1 or ING2 to induce cellular 
senescence in transformed cancer cells, such as by re-expression, are not yet clear.

The molecular pathway to induce cellular senescence is also under investigations. A functional link of both ING isoforms has also been reported to increase $\mathrm{p} 53$ protein stability by posttranslational modification that enhances the transcriptional activity of p53 and thereby triggering the cellular senescence phenotype [34,51,52]. However, it seems that both ING1 and ING2 can trigger the cellular senescence via more than one pathway by revealing also a p53independent pathway of ING-mediated cellular senescence $[36,53]$ as well as via the p16-pRb pathway [49] (Figure 1).

Notably, the PHD region plays an essential role to induce cellular senescence. Human fibroblasts transfected with PHD mutants of ING1 with a deficient histone binding ability were not capable to undergo cellular senescence [54], thus supports the important role of PHD and histone binding of ING proteins for the induction of cellular senescence. This finding strongly links the epigenetic regulation of ING tumor suppressors with cellular senescence induction also in nontumor cells.

\section{The ING - PHD: a highly conserved ING-domain between plants, insects and mammals}

The ING PHD domain is relevant for both epigenetic control and induction of cellular senescence. Interestingly, the human ING1b and ING2a proteins are not only sharing high homologies in their amino acid sequences, but is also found to have high amino acid homology to ING proteins of other species, especially in the PHD region (Figure 2).

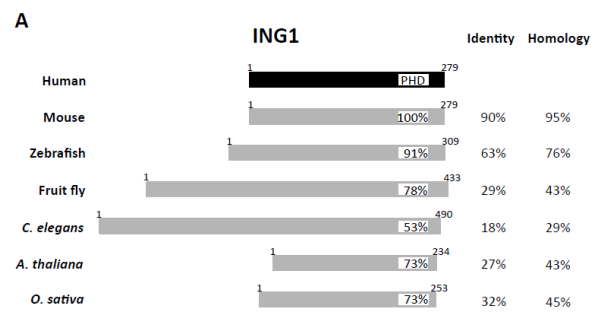

Figure 2A: Conserved amino acid sequence of ING1 proteins.

(A) Human ING1b (NCBI ref: NP_937862.1) is aligned to mouse ING1 (NP_036049.2), zebrafish Ing1 (NP_001035446.1), fruit fly ING (NP_650656.1), C. elegans ING homolog (NP_496909.1), A. thaliana ING1 (NP_566742.1), and $O$. sativa PHD finger protein ING (NP_001048939.1).

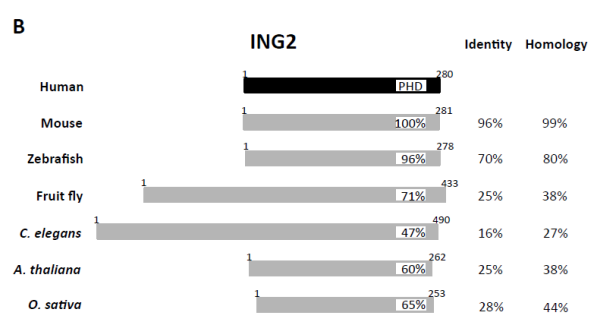

Figure 2B: Conserved amino acid sequence of ING2 proteins.
(B) Human ING2a (NP_001555.1) is aligned to mouse ING2 (NP_075992.2), zebrafish Ing2 (NP_001002448.1), fruit fly ING (NP_650656.1), C. elegans ING homolog (NP_496909.1), A. thaliana ING2 (NP_974026.1), and O. sativa PHD finger protein ING (NP_001048939.1).

The NCBI BLAST program (http://blast.ncbi.nlm.nih.gov) was used to identify the most homologous proteins to human ING1b or human ING2a in other species. In addition, the whole protein alignment between human INGs and other species were performed with EMBOSS needle program (http://www.ebi.ac.uk/Tools/) to calculate the percentage identity and homology of amino acid sequences. The amino acid positions of each protein are indicated. The plant homeodomain (PHD) region of each species is separately aligned and compared as the percentage identity to the human PHD. Among other isoforms in D. melanogaster (fruit fly), C. elegans and $O$. sativa, the identified ING homologs exhibit the highest similarity to both human ING1 and ING2.

In other species including mouse (Mus musculus), zebrafish (Danio reio), fruit fly (D. melanogaster), nematode (C. elegans), and plant (Arabidopsis thaliana and Oryza sativa) ING homologues were identified. Among these species, the mouse ING1 (NCBI ref: NP_036049.2) and ING2 (NP_075992.2) proteins share the highest identity (90\% and 96\%) and homology (95\% and 99\%) of amino acid sequences to the human ING1b (NP_937862.1) and ING2a (NP_001555.1) proteins, respectively (Figure 2). Notably, both PHD amino acid sequences of ING1 and ING2 from mouse ING proteins are $100 \%$ identical to PHD of human ING proteins indicating an important function. This is further supported by the fact that more than $50 \%$ of the amino acid sequences in the PHD region of plants ( $A$. thaliana and $O$. sativa) INGs are identical with the human PHD of INGs. Of note, the plants and fruit fly PHD domains share higher identity to human PHD as compared to the PHD region of $C$. elegans (Figure 2). The protein alignments suggest that ING proteins and their PHD seem to be under strong evolutional selection and therefore, existing and being conserved in many species.

\section{Phenotype of genetic ING models in vivo}

The role of ING factors in vivo was analyzed using mice knock-out models. Despite the high homology of ING factors between plants and mammals, which implies an important functional role, a relatively weak phenotype in the $\mathrm{KO}$ mice was surprising. $\mathrm{KO}$ mice of either ING1 or ING2 were viable but promote tumor development $[36,37,55]$. ING1 KO mice revealed a high incidence of $\mathrm{B}$ cell lymphoma development $[36,55]$. ING2 KO mice on the other hand, were observed with the development of soft-tissue sarcomas [37]. Of note, the male ING2 KO mice exhibited the particular phenotype of being infertile and having small testes. These mice showed deficient spermatogenesis, altered meiotic recombination, and failed to complete meiosis II [37]. Interestingly, ING2 also seems to play a role in preimplantation development [56]. The ING2 expression was observed to be rapidly increased during the 2 -cell to 4 -cell cleavage-stage. In line with this, KD of ING2 in mouse zygote slows down the embryonic development [56].

The relatively mild phenotype of $\mathrm{KO}$ mice of these highly conserved factors suggests that ING1 and ING2 share similar functions and may compensate for the loss of function of the ING2 or ING1 null mutant, respectively. Also other ING family members might take over some essential functions for null mutants of one $I N G$ gene. Thus, we propose that the presence of multiple $I N G$ genes might serve as a redundant 
and security viability system to reduce disadvantageous mediated by mutations of one of the $I N G$ genes.

Similarly, in $C$. elegans the depletion of the ING homolog protein suggests that this protein inhibits ionizing radiation-induced germ-cell apoptosis [57]. Moreover, nematodes expressing a mutant ING protein exhibit a weak uncoordinated phenotype.

Although ING proteins are rarely studied in plants, the functions and effects of PHD fingers of other factors are well established. Many proteins in plant contain putative PHD fingers and were found to be involved in various developmental processes including flowering, development of anthers, and inflorescences [58-60].

ING factors epigenetically regulate the gene expression of both mRNA and miRNA genes $[42,50,61,62]$. However, not much is known about the (I) control of binding to ING interacting factors, (II) the ING-specific transcriptome landscape, (III) mechanism(s) that control normal cell cycle by ING isoforms, (IV) sensing of cellular stress and its signaling that affects ING-factors to induce cellular senescence, apoptosis or DNA repair. Further it is unclear (V) which of the ING isoform functions is lost or decreased during early tumorigenesis.

Also the functional overlapping role of tumor suppressive function by each ING factor is unclear. However, there must be important biological reasons for the ING proteins to be naturally selected with a very high preservation of their amino acid sequence. Thus, many questions remain open to better understand the epigenetics of ING pathway as tumor suppressors and with relevance to human aging.

\section{Acknowledgement}

We are grateful to Dr. Mohsen Esmaeili and Tim Schmaeche for critically reading the manuscript.

\section{References}

1. Zahn JM, Poosala S, Owen AB, Ingram DK, Lustig A, et al. (2007) AGEMAP: a gene expression database for aging in mice. PLoS Genet 3: e201.

2. Greer EL, Maures TJ, Ucar D, Hauswirth AG, Mancini E, et al. (2011) Transgenerational epigenetic inheritance of longevity in Caenorhabditis elegans. Nature 479: 365-371.

3. Wood JG, Hillenmeyer S, Lawrence C, Chang C, Hosier S, et al. (2010) Chromatin remodeling in the aging genome of Drosophila. Aging Cell 9: 971-978.

4. Gami MS, Wolkow CA (2006) Studies of Caenorhabditis elegans DAF-2/ insulin signaling reveal targets for pharmacological manipulation of lifespan. Aging Cell 5: 31-37.

5. Tatar M, Kopelman A, Epstein D, Tu MP, Yin CM, et al. (2001) A mutant Drosophila insulin receptor homolog that extends life-span and impairs neuroendocrine function. Science 292: 107-110.

6. McGue M, Vaupel JW, Holm N, Harvald B (1993) Longevity is moderately heritable in a sample of Danish twins born 1870-1880. J Gerontol 48: B237-244.

7. Herskind AM, McGue M, Holm NV, Sørensen TI, Harvald B, et al. (1996) The heritability of human longevity: a population-based study of 2872 Danish twin pairs born 1870-1900. Hum Genet 97: 319-323.

8. Gavrilova NS, Gavrilov LA, Evdokushkina GN, Semyonova VG, Gavrilova AL, et al. (1998) Evolution, mutations, and human longevity: European royal and noble families. Hum Biol 70: 799-804.

9. Cournil A, Legay JM, Schächter F (2000) Evidence of sex-linked effects on the inheritance of human longevity: a population-based study in the Valserine valley (French Jura), 18-20th centuries. Proc Biol Sci 267: 1021-1025.
10. Cournil A, Kirkwood TB (2001) If you would live long, choose your parents well. Trends Genet 17: 233-235.

11. Berger SL, Kouzarides T, Shiekhattar R, Shilatifard A (2009) An operational definition of epigenetics. Genes Dev 23: 781-783.

12. Benayoun BA, Pollina EA, Brunet A, et al. (2015) Epigenetic regulation of ageing: linking environmental inputs to genomic stability. Nat Rev Mol Cell Biol 16: 593-610.

13. Brunet A, Berger SL (2014) Epigenetics of aging and aging-related disease. J Gerontol A Biol Sci Med Sci 69 Suppl 1: S17-20.

14. Halaschek-Wiener J, Amirabbasi-Beik M, Monfared N, Pieczyk M, Sailer C, et al. (2009) Genetic variation in healthy oldest-old. PLoS One 4: e6641.

15. Tallen G, Riabowol K (2014) Keep-ING balance: tumor suppression by epigenetic regulation. FEBS Lett 588: 2728-2742.

16. Doyon Y, Cayrou C, Ullah M, Landry AJ, Côté V, et al. (2006) ING tumor suppressor proteins are critical regulators of chromatin acetylation required for genome expression and perpetuation. Mol Cell 21: 51-64.

17. Hayflick L, Moorhead PS (1961) The serial cultivation of human diploid cell strains. Exp Cell Res 25: 585-621.

18. Muñoz-Espín D, Cañamero M, Maraver A, Gómez-López G, Contreras J, et al. (2013) Programmed cell senescence during mammalian embryonic development. Cell 155: 1104-1118.

19. Collado M, Serrano M (2010) Senescence in tumours: evidence from mice and humans. Nat Rev Cancer 10: 51-57.

20. Prieur A, Peeper DS (2008) Cellular senescence in vivo: a barrier to tumorigenesis. Curr Opin Cell Biol 20: 150-155.

21. Campisi J (2013) Aging, cellular senescence, and cancer. Annu Rev Physiol 75: 685-705.

22. Goeman F, Thormeyer D, Abad M, Serrano M, Schmidt O, et al. (2005) Growth inhibition by the tumor suppressor p33ING1 in immortalized and primary cells: involvement of two silencing domains and effect of Ras. Mol Cell Biol 25: 422-431.

23. Pedeux R, Sengupta S, Shen JC, Demidov ON, Saito S, et al. (2005) ING2 regulates the onset of replicative senescence by induction of $\mathrm{p} 300$ dependent p53 acetylation. Mol Cell Biol 25: 6639-6648.

24. He GH, Helbing CC, Wagner MJ, Sensen CW, Riabowol K (2005) Phylogenetic analysis of the ING family of PHD finger proteins. Mol Biol Evol 22: 104-116.

25. Nouman GS, Anderson JJ, Wood KM, Lunec J, Hall AG, et al. (2002) Loss of nuclear expression of the p33(ING1b) inhibitor of growth protein in childhood acute lymphoblastic leukaemia. J Clin Pathol 55: 596-601.

26. Oki E, Maehara Y, Tokunaga E, Kakeji Y, Sugimachi K (1999) Reduced expression of $\mathrm{p} 33$ (ING1) and the relationship with p53 expression in human gastric cancer. Cancer Lett 147: 157-162.

27. Borkosky SS, Gunduz M, Nagatsuka H, Beder LB, Gunduz E, et al. (2009) Frequent deletion of ING2 locus at 4q35.1 associates with advanced tumor stage in head and neck squamous cell carcinoma. J Cancer Res Clin Oncol 135: 703-713.

28. Zhang HK, Pan K, Wang H, Weng DS, Song HF, et al. (2008) Decreased expression of ING2 gene and its clinicopathological significance in hepatocellular carcinoma. Cancer Lett 261: 183-192.

29. Walzak AA, Veldhoen N, Feng X, Riabowol K, Helbing CC (2008) Expression profiles of mRNA transcript variants encoding the human inhibitor of growth tumor suppressor gene family in normal and neoplastic tissues. Exp Cell Res 314: 273-285.

30. Ythier D, Brambilla E, Binet R, Nissou D, Vesin A, et al. (2010) Expression of candidate tumor suppressor gene ING2 is lost in non-small cell lung carcinoma. Lung Cancer 69: 180-186.

31. Toyama T, Iwase H, Watson P, Muzik H, Saettler E, et al. (1999) Suppression of ING1 expression in sporadic breast cancer. Oncogene 18: 5187-5193.

32. Guérillon C, Larrieu D, Pedeux R (2013) ING1 and ING2: multifaceted tumor suppressor genes. Cell Mol Life Sci 70: 3753-3772. 
33. Garkavtsev I, Kazarov A, Gudkov A, Riabowol K (1996) Suppression of the novel growth inhibitor p33ING1 promotes neoplastic transformation. Nat Genet 14: 415-420.

34. Nagashima M, Shiseki M, Miura K, Hagiwara K, Linke SP, et al. (2001) DNA damage-inducible gene p33ING2 negatively regulates cell proliferation through acetylation of p53. Proc Natl Acad Sci U S A 98: 9671-9676.

35. Garkavtsev I, Riabowol K (1997) Extension of the replicative life span of human diploid fibroblasts by inhibition of the p33ING1 candidate tumor suppressor. Mol Cell Biol 17: 2014-2019.

36. Coles AH, Liang H, Zhu Z, Marfella CG, Kang J, et al. (2007) Deletion of p37Ing1 in mice reveals a p53-independent role for Ing1 in the suppression of cell proliferation, apoptosis, and tumorigenesis. Cancer Res 67: 2054-2061.

37. Saito M, Kumamoto K, Robles AI, Horikawa I, Furusato B, et al. (2010) Targeted disruption of Ing2 results in defective spermatogenesis and development of soft-tissue sarcomas. PLoS One 5: e15541.

38. Ythier D, Larrieu D, Brambilla C, Brambilla E, Pedeux R (2008) The new tumor suppressor genes ING: genomic structure and status in cancer. Int J Cancer 123: 1483-1490.

39. Ludwig S, Klitzsch A, Baniahmad A (2011) The ING tumor suppressors in cellular senescence and chromatin. Cell Biosci 1: 25.

40. Santos-Rosa H, Schneider R, Bannister AJ, Sherriff J, Bernstein BE, et al. (2002) Active genes are tri-methylated at K4 of histone H3. Nature 419: 407-411.

41. Skowyra D, Zeremski M, Neznanov N, Li M, Choi Y, et al. (2001) Differential association of products of alternative transcripts of the candidate tumor suppressor ING1 with the mSin3/HDAC1 transcriptional corepressor complex. J Biol Chem 276: 8734-8739.

42. Shi X, Hong T, Walter KL, Ewalt M, Michishita E, et al. (2006) ING2 PHD domain links histone $\mathrm{H} 3$ lysine 4 methylation to active gene repression. Nature 442: 96-99.

43. Goeman F, Otto K, Kyrylenko S, Schmidt O, Baniahmad A (2008) ING2 recruits histone methyltransferase activity with methylation site specificity distinct from histone H3 lysines 4 and 9. Biochim Biophys Acta 1783: 1673-1680.

44. Vieyra D, Loewith R, Scott M, Bonnefin P, Boisvert FM, et al. (2002) Human ING1 proteins differentially regulate histone acetylation. J Biol Chem 277: 29832-29839.

45. Schäfer A, Karaulanov E, Stapf U, Döderlein G, Niehrs C (2013) Ing1 functions in DNA demethylation by directing Gadd45a to H3K4me3. Genes Dev 27: 261-273.

46. Cheung KJ Jr, Mitchell D, Lin P, Li G (2001) The tumor suppressor candidate p33(ING1) mediates repair of UV-damaged DNA. Cancer Res 61: 4974-4977.

47. Scott M, Bonnefin P, Vieyra D, Boisvert FM, Young D, et al. (2001) UVinduced binding of ING1 to PCNA regulates the induction of apoptosis. J Cell Sci 114: 3455-3462.

48. Larrieu D, Ythier D, Binet R, Brambilla C, Brambilla E, et al. (2009) ING2 controls the progression of DNA replication forks to maintain genome stability. EMBO Rep 10: 1168-1174.
49. Li N, Li Q, Cao X, Zhao G, Xue L, et al. (2011) The tumor suppressor p33ING1b upregulates p16INK4a expression and induces cellular senescence. FEBS Lett 585: 3106-3112.

50. Larrieu D, Ythier D, Brambilla C, Pedeux R (2010) ING2 controls the G1 to S-phase transition by regulating p21 expression. Cell Cycle 9: 3984-3990.

51. Garkavtsev I, Grigorian IA, Ossovskaya VS, Chernov MV, Chumakov PM, et al. (1998) The candidate tumour suppressor p33ING1 cooperates with p53 in cell growth control. Nature 391: 295-298.

52. Atadja P, Wong H, Garkavtsev I, Veillette C, Riabowol K (1995) Increased activity of p53 in senescing fibroblasts. Proc Natl Acad Sci U S A 92: 8348-8352.

53. Kumamoto K, Spillare EA, Fujita K, Horikawa I, Yamashita T, et al. (2008) Nutlin-3a activates p53 to both down-regulate inhibitor of growth 2 and up-regulate mir-34a, mir-34b, and mir-34c expression, and induce senescence. Cancer Res 68: 3193-3203.

54. Abad M, Moreno A, Palacios A, Narita M, Blanco F, et al. (2011) The tumor suppressor ING1 contributes to epigenetic control of cellular senescence. Aging Cell 10: 158-171.

55. Kichina JV, Zeremski M, Aris L, Gurova KV, Walker E, et al. (2006) Targeted disruption of the mouse ing1 locus results in reduced body size, hypersensitivity to radiation and elevated incidence of lymphomas. Oncogene 25: 857-866.

56. Zhou L, Wang P, Zhang J, Heng BC, Tong GQ (2016) ING2 (inhibitor of growth protein-2) plays a crucial role in preimplantation development. Zygote 24: 89-97.

57. Luo J, Shah S, Riabowol K, Mains PE (2009) The Caenorhabditis elegans ing-3 gene regulates ionizing radiation-induced germ-cell apoptosis in a p53-associated pathway. Genetics 181: 473-482.

58. Matsubara K, Yamanouchi U, Nonoue Y, Sugimoto K, Wang ZX, et al. (2011) Ehd3, encoding a plant homeodomain finger-containing protein, is a critical promoter of rice flowering. Plant J 66: 603-612.

59. Li H, Yuan Z, Vizcay-Barrena G, Yang C, Liang W, et al. (2011) PERSISTENT TAPETAL CELL1 encodes a PHD-finger protein that is required for tapetal cell death and pollen development in rice. Plant Physiol 156: 615-630.

60. Choi SC, Lee S, Kim SR, Lee YS, Liu C, et al. (2014) Trithorax group protein Oryza sativa Trithorax1 controls flowering time in rice via interaction with early heading date3. Plant Physiol 164: 1326-1337.

61. Takahashi M, Seki N, Ozaki T, Kato M, Kuno T, et al. (2002) Identification of the p33(ING1)-regulated genes that include cyclin B1 and proto-oncogene DEK by using cDNA microarray in a mouse mammary epithelial cell line NMuMG. Cancer Res 62: 2203-2209.

62. Chen J, Tran UM, Rajarajacholan U, Thalappilly S, Riabowol K (2013) ING1b-inducible microRNA203 inhibits cell proliferation. Br J Cancer 108: 1143-1148. 